\title{
Crosslayer Survivable Mapping in Overlay-IP-WDM Networks
}

\author{
Peera Pacharintanakul and David Tipper \\ Graduate Telecommunications and Networking Program, \\ University of Pittsburgh, Pittsburgh, PA 15260, United States \\ Email: \{peerap,tipper\}@ tele.pitt.edu
}

\begin{abstract}
Survivability is a critical requirement for reliable services in any network. As the Internet moves towards a three layer architecture consisting of Overlay on top of IP on top of WDM-based networks incorporating the interaction between and among network layers is crucial for efficient and effective implementation of survivability. This paper highlights the challenges of providing survivability in three-layer networks and develops a novel approach crosslayer survivable mapping to offer such survivability in an efficient way. We investigate the impacts of overlay network dependency on lower layer network layout in terms of the capacity allocated to overlay paths. The results demonstrate the impact due to layer dependency that it is more severe than initially anticipated making it clear that single layer network design is inadequate to assure service guarantees and efficient capacity allocation.
\end{abstract}

Index Terms - Crosslayer survivable mapping, Joint capacity allocation, Multilayer networks, Survivability

\section{INTRODUCTION}

The phenomenal growth of the Internet is partly explained by the proliferation of underlying optical WDM technologies and overlaying applications. Research along these three directions may not yet be converged, but evidences justify such emergence are leading us to consider a network of three layers, Overlay-over-IP-over-WDM, as the future Internet, which might otherwise not have been considered.

In this architecture, overlay nodes are attached to an IP router. IP routers are associated with an optical WDM switch; the switches are then interconnected by multi-wavelength fibers capable of carrying a number of transmission channels. Recognizing the lower two network layers, IP and WDM, each IP route is established by one lightpath or more that spans across fibers and occupies one wavelength in each fiber. These two layers constitute the Internet backbone in the optical Internet. Dependency of failures, routing, topology distribution, and signaling protocols between the two layers are still major concerns; however, thanks to the vertical integration of IP and WDM technologies in the backbone network, these two-layer networks can now be supported by GMPLS mechanisms acting as a glue layer of the two technology environments.

Failures at the IP layer may occur for several reasons. One of them is related to failures at the WDM optical layer such as node or link failures; an optical-related failure can

Financial support to Peera Pacharintanakul through a TOT Graduate Fellowship, Thailand, is gratefully acknowledged. bring down many failures at the IP layer. This effect is called failure propagation, the severity of which is partly because of improper IP-to-WDM link mapping. Such a link mapping tells us on which of the lightpaths an IP path is routed. Reference [1] reveals that, in a highly-meshed operational IP network, this ill-chosen mapping contributes to $12 \%$ of all unplanned failures that affect the IP traffic. The figure is likely to be higher in partial-meshed IP networks as it increases the chance of network partitioning or reduces the number of rerouting choices when a backup path is needed or failures happen. More interestingly, the authors also discover that, on average, 8 WDM links and 7 nodes are shared by failed IP paths. In such circumstances the need for good link mapping to mitigate the worst consequences of failures is accepted.

At the WDM layer, survivability is normally achieved through redundant paths - a number of backup paths and a primary path. Dedicated protection, $1+1$, allows one backup path to protect the primary path by simultaneously sending the same traffic on both paths. Alternatively, shared protection, $1: \mathrm{N}$, allows the backup path to be shared by $\mathrm{N}$ primary paths. However, in this case, no more than one primary path can fail at any given time as the only backup path has to be shared. Shared-backup path protection (SBPP) allows capacity of each backup path protecting a primary path to be shared; therefore, the survivability can be achieved with the least spare capacity requirement. These protection schemes can be applied either at a lightpath level or line level. By protecting at a lightpath level - optical channel section (OCh-S) - each failed lightpath can be restored individually at a failure time. Alternatively, line level protection - optical multiplex section (OMS) - restores an entire group of the lightpaths that belong to a failed link without allowing for individual recovery [2].

There has been a number of survivability strategies proposed to recover from failures in a network of the two layers. These strategies primarily lie in the choices of 1) in which layer the strategy is implemented (top (IP), bottom (WDM), both, none); 2) primary-backup paths disjointness (full, partial); 3) coordination of the two layers (coordinated, uncoordinated); 4) resource of failed paths, whether it can be reassigned to a newly calculated backup path (released, held); and, 5) when to compute a backup path (preplanned, upon failures), etc. In the case of implementing the survivability strategy at both layers, two extended choices are also needed. First, which layer is to 
recover from failures first. It is called a bottom-up escalation strategy if the WDM layer is to take responsibility for failure recovery before the IP layer and a top-down strategy if they are reversed in order. Second, when that layer is to transfer its responsibility to the other. The decision can be based on either a timeout value or a token; however, it is further required that both layers are notified when failures happen. References [3] - [9] provide discussions on the survivability strategies in two-layer networks in more detail.

In contrast to the IP-over-WDM architecture, an overlay network is normally formed without a knowledge of the underlying two layers. This can be a problem when failures happen. While high survivability is usually achieved by a number of redundant paths that are disjoint at the overlay layer, they may not also be necessarily disjoint at the physical WDM layer. Reference [10] proposes two survivability models for an overlay network. One permits node disjointness at the WDM layer in all redundant paths whereas the other permits only a guaranteed number of the paths that are node disjoint. Unfortunately, they both are NP-complete. Furthermore, this proposal requires an overlay network to have topology knowledge of the underlying networks. One other direction is for the overlay to take complete responsibility for failures at the lower layers. Resilient overlay networks [11] (RONs) architecture, a well-known solution in this direction, aims at recovering from failures by forming a complete graph at the overlay layer over the IP layer; these networks can provide customized path selections specific to each application; however, they still need to be aware of the underlying IP/WDM networks, as duplicate transmissions in the physical link may occur due to improper mapping. Although overlay nodes are often referred to end hosts, this paper considers more long-lived nodes, which are commonly called supernodes, gateways, or computer servers, that tend to be more stable as our interest is core backbone networks. For all these reasons, survivability by crosslayer mapping is a major concern for layered networks.

There are some current planning approaches that mention networks with three layers, for example, ATM-over-SDHover-WDM [4], [12] or IP/MPLS-over-WDM [13]; however, they either consider each pair of the two sublayers at a time or view them as a single super layer. In this paper, we explicitly consider an Overlay-over-IP-over-WDM network as an instance of three-layer networks and address survivability from the viewpoint of the mapping in the core backbone network.

The rest of the paper is organized as follows: Section II presents the routing problem in a network of three layers. Section III defines the problem and proposes a mathematical model to provide minimum joint primary-backup capacity allocation for survivability of the traffic that originates at each and every network layer. Evaluation results and analysis are provided in Section IV. Finally, our conclusions are given in Section V.

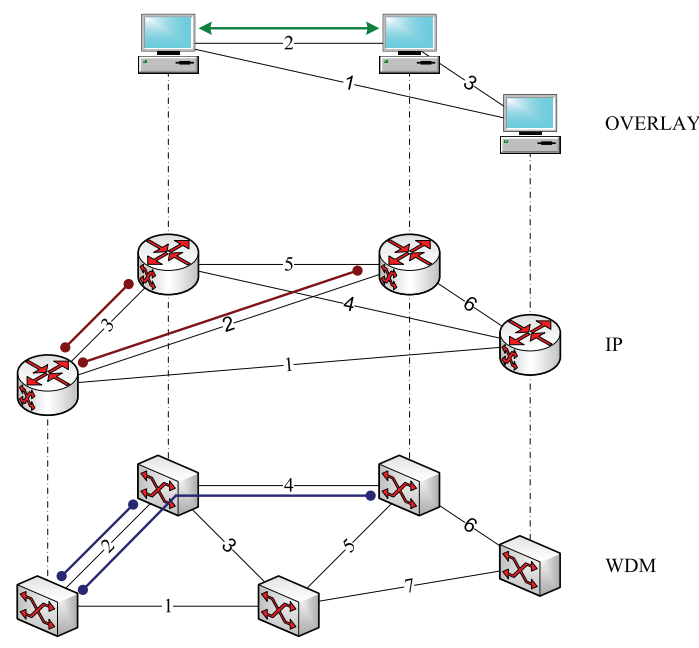

Fig. 1. Backhaul routing in a three-layer network

\section{BACKhaul Routing IN ThreE-LAyer Networks}

Existing survivable mapping, which is also known as survivable routing or logical topology planning either explicitly considers two-layer topology such as IP-over-WDM or deals with a logical topology constrained within a physical network such as WDM lightpaths in an optical fiber cable [14].

However, overlay networks are receiving more attention not only because they can offer new services but also for the reason that they can overcome functionality limitations of the Internet without the need for modifications of the underlying network layers; therefore, it is important that this overlay network layer also be included in the network design to complete the whole picture.

In order to understand how layers interact, in the following paragraph, we systematically present one property which is critical in designing highly survivable overlay networks and services on top of the two-layer network, IP-over-WDM.

Consider the sample network illustrated in Fig. 1. Here, the overlay, IP, and WDM networks consist of 3 nodes, 3 links, 4 nodes, 6 links, and 5 nodes, 7 links, respectively. The numbers on each link indicate the link index. In order to achieve routing that is survivable to any underlying single link failure in both two-layer networks, we need to ensure that each mapping is at least two-connected [14]. Let $H_{i}^{j}: \mathcal{L}^{(j)} \mapsto \mathcal{L}^{(i)}$ be a link mapping of Layer $j$ onto Layer $i$ where each $j$-th layer link is assigned to a subset of $i$-th layer links. When put in a matrix form, the mapping has rows corresponding to layer $j$ links whereas columns corresponding to layer $i$ links. Such mappings according to the figure can be given as

$$
H_{2}^{3}=\begin{aligned}
& 1 \\
& 2 \\
& 3
\end{aligned}\left[\begin{array}{llllll}
1 & 2 & 3 & 4 & 5 & 6 \\
0 & 0 & 0 & 1 & 0 & 0 \\
0 & 1 & 1 & 0 & 0 & 0 \\
0 & 0 & 0 & 0 & 0 & 1
\end{array}\right]
$$

for the Overlay-over-IP, i.e., Layer 3 to Layer 2, network and 


$$
H_{1}^{2}=\begin{aligned}
& 1 \\
& 2 \\
& 3 \\
& 4 \\
& 5 \\
& 6
\end{aligned}\left[\begin{array}{lllllll}
1 & 2 & 3 & 4 & 5 & 6 & 7 \\
1 & 0 & 0 & 0 & 0 & 0 & 1 \\
0 & 1 & 0 & 1 & 0 & 0 & 0 \\
0 & 1 & 0 & 0 & 0 & 0 & 0 \\
0 & 0 & 1 & 0 & 0 & 0 & 1 \\
0 & 0 & 0 & 1 & 0 & 0 & 0 \\
0 & 0 & 0 & 0 & 0 & 1 & 0
\end{array}\right]
$$

for the IP-over-WDM network, i.e., Layer 2 to Layer 1, network.

While this routing is survivable, it may not also be resourceefficient or loop-free. Consider link 2 at the overlay layer. This link is routed on links 2 and 3 at the IP layer. However, because these IP links 2 and 3 use links 2 and 4 , and 2 at the WDM layer, respectively, the WDM link 2 is used twice; thus, the solution ref. [14] proposes to ensure survivability as well as other solutions in the current literature can be resource inefficient. In this paper, we consider survivable mapping such that no such backhaul occurs.

\section{PRoblem Statement}

In this work, we consider the survivability problem in threelayer networks such that no backhaul occurs and survivability is defined by SBPP under single-link failures.

\section{A. Notation Used}

The notation used in this paper can be summarized as follows:

$l \quad$ Network layer $l$, where $l=1,2,3$ refers to WDM, IP, and Overlay layer, respectively

$\mathcal{N}^{(l)} \quad$ l-th layer node set

$\mathcal{L}^{(l)} \quad l$-th layer link set

$F^{(l)} \quad l$-th layer flow vector

$M^{(l)} \quad$ Diagonal matrix of bandwidth of $l$-th layer flow $f^{(l)}$ with dimension $\left|F^{(l)}\right| \times\left|F^{(l)}\right|$

$B^{(l)} \quad l$-th layer incidence matrix with dimension $\left|\mathcal{N}^{(l)}\right| \times\left|\mathcal{L}^{(l)}\right|$

$D^{(l)} \quad l$-th layer flow-node incidence matrix with dimension $\left|F^{(l)}\right| \times\left|\mathcal{N}^{(l)}\right|$

$P^{(l)} \quad l$-th layer primary path matrix with dimension $\left|F^{(l)}\right| \times\left|\mathcal{L}^{(l)}\right|$

$Q^{(l)} \quad l$-th layer backup path matrix with dimension $\left|F^{(l)}\right| \times\left|\mathcal{L}^{(l)}\right|$

$W^{(l)} \quad l$-th layer primary-path link capacity vector with dimension $\left|\mathcal{L}^{(l)}\right| \times 1$

$G^{(l)} \quad l$-th layer spare capacity matrix with dimension $\left|\mathcal{L}^{(l)}\right| \times\left|\mathcal{L}^{(l)}\right|$

$S^{(l)} \quad l$-th layer backup-path link capacity vector with dimension $\left|\mathcal{L}^{(l)}\right| \times 1$

$I \quad$ Identity matrix

$H_{i}^{j} \quad$ Survivable mapping matrix between Layers $j$ and $i\left(H_{0}^{1}=I\right)$

e The column vector of all $1 \mathrm{~s}$ in $\mathbb{R}^{n}$
In addition, we consider the following mapping notation specific to the IP-over-WDM networks in our considered threelayer networks.

$$
\begin{aligned}
B^{\left(2^{\prime}\right)} & =B^{(2)} \\
D^{\left(2^{\prime}\right)} & =\left[D^{(2)} \mid 0\right] \\
P^{\left(2^{\prime}\right)} & =P^{(2)} H_{1}^{2} \\
M^{\left(2^{\prime}\right)} & =M^{(2)}
\end{aligned}
$$

Because the incidence matrix is topology dependent, a Layer 2 incidence matrix, when mapped to Layer 1 , is equivalent to that of Layer 1. This is shown in eq. (1) by the prime $\left({ }^{\prime}\right)$ notation. Since a Layer 2 network may not have the same number of nodes as the Layer 1, the flow-node incidence matrix in (2) is mapped to Layer 1 by padding columns of zeros as necessary to $D^{(2)}$. Equation (3) computes the primary paths of Layer 2 from the perspective of Layer 1 given as $P^{\left(2^{\prime}\right)}$ using the link-mapping information in $H_{1}^{2}$. In (4), the bandwidth of the mapped 2-nd layer flow still holds the same value as that of the 2-nd layer flow as it depends on flows - in terms of both bandwidths and connections - only. The motivation behind these equations will be briefly discussed in the next section; its details are given in [15].

\section{B. Problem Formulation}

The $l$-th layer topology of an uncapacitated network consists of a set of nodes $\mathcal{N}^{(l)}$ and a set of bidirectional links $\mathcal{L}^{(l)}$ Each link is equivalent to two unidirectional links with an equal number of wavelengths and the same failure state, i.e., available or failed, in each link. Then the incidence matrix $B^{(l)}$ [16] can be obtained.

Traffic originating at Layer $l$ can be formulated as follows: Let $D^{(l)}$ denote a flow-node incidence matrix at Layer $l$ in which each row represents a source-sink pair of a flow $f^{(l)}$ where $1 \leq f^{(l)} \leq\left|F^{(l)}\right|$ and $F^{(l)}$ is a vector of flow at Layer $l$. The matrix $D^{(\bar{l})}$ has +1 in the row corresponding to a traffic source and -1 in the row corresponding to a traffic sink. Flow bandwidth is integral and is represented by $m^{(l)}\left(f^{(l)}\right)$ for each flow $f^{(l)}$. The bandwidth matrix $M^{(l)}$ puts $m^{(l)}\left(f^{(l)}\right)$ on the main diagonal; therefore, this matrix is a diagonal matrix with dimension $\left|F^{(l)}\right| \times\left|F^{(l)}\right|$. Identical source-sink pairs are here regarded as a single flow of all bandwidths combined.

Using the network information given previously, survivability using SBPP scheme at Layer $l$ can be found as follows: Let $P^{(l)}$ be the primary path matrix with dimension $\left|F^{(l)}\right| \times\left|\mathcal{L}^{(l)}\right|$. For the element $p_{f e}^{(l)}$ of $P^{(l)}, p_{f e}^{(l)}=1$ if link $e$ is in the primary path of flow $f^{(l)}$, and 0 otherwise. Likewise, the backup path matrix $Q^{(l)}$ with the same dimension as the primary path can be found in a similar manner.

In order to route a link in Layer $l+1$ on Layer $l$ properly, a good link mapping such that the $(l+1)$-th layer topology remains connected even after a single link failure in Layer $l$ is required. Such a mapping is given in [14] and is formulated as a mathematical model in [17]; however, an additional requirement is needed in networks consisting of three layers 
or more to prevent backhaul. This requirement is given as follows:

Definition 1. The condition $C_{\mathcal{L}}$ of the link mapping in threelayer networks such that no backhaul routing occurs is defined by: $H_{2}^{3} H_{1}^{2} \preceq 1$, where the $\preceq$ symbol denotes element-wise comparison.

The following Lemma is motivated by the condition $C_{\mathcal{L}}$.

Lemma 1 (Crosslayer Mapping). No backhaul routing occurs in a three-layer network if and only if a link mapping satisfies the condition $C_{\mathcal{L}}$.

Proof: Given two survivable mappings, $H_{2}^{3}$ and $H_{1}^{2}$, in a three-layer network, it can be followed that an element $(i, j)$ of the matrix product $H_{2}^{3} H_{1}^{2}$ is nonzero if and only if a Layer 1 link $j$ is used by a Layer 3 link $i$. This element determines the number of times Layer 1 link $j$ is used by Layer 3 link $i$. Because in no backhaul routing there should be exactly one such use by any path in Layer 3 ; therefore, the condition $C_{\mathcal{L}}$ : $H_{2}^{3} H_{1}^{2} \preceq 1$ is required in routing with no backhaul.

Clearly, the proof of Lemma 1 constructs efficient routing in three-layer networks. Next, we show that the situation is much more favorable when survivability is also considered.

Theorem 1 (Crosslayer Survivable Mapping). A routing is survivable and produces no backhaul if and only if its corresponding link mapping is two-connected and satisfies the condition $C_{\mathcal{L}}$.

Proof: Necessity and Sufficiency are a direct result of [14, Theorem 1] and Lemma 1 previously given.

In addition, it can also be seen that the mapping can withstand multiple link failures if proper survivable mapping ( [18], [19]) is employed.

The formulation of the Overlay-over-IP-over-WDM networks can be summarized in a mathematical model as follows: Denote $l=1,2,3$ as the WDM, IP, and Overlay layer, respectively; this alpha numeric notation may be used interchangeably in this section as well as throughout the paper. The joint capacity allocation (JCA) problem in three-layer networks (JCA3L) can be formulated as follows:

Objective:

$$
\min _{P^{(l)}, Q^{(l)}} \sum_{l=\{1,2,3\}}\left(W^{(l)^{T}} \prod_{k=1}^{l} H_{k-1}^{k}\right) \mathbf{e}+\sum_{l=\left\{1,2^{\prime}, 3\right\}} S^{(l)}{ }^{T} \mathbf{e},
$$

s.t.

Primary Paths:

$$
\begin{aligned}
P^{(l)} B^{(l)}{ }^{T} & =D^{(l)}, & & l \in\{1,2,3\} \\
W^{(l)} & =\left(M^{(l)} P^{(l)}\right)^{T} \mathbf{e}, & & l \in\{1,2,3\} \\
W^{(l)} & \in \mathbb{R}^{\left|\mathcal{L}^{(l)}\right|}, & & l \in\{1,2,3\} \\
P^{(l)} & : \text { binary matrix, } & & l \in\{1,2,3\}
\end{aligned}
$$

Backup Paths:

$$
\begin{aligned}
Q^{(l)} B^{(l)^{T}} & =D^{(l)}, & & l \in\left\{1,2^{\prime}, 3\right\} \\
Q^{(l)} & : \text { binary matrix, } & & l \in\left\{1,2^{\prime}, 3\right\}
\end{aligned}
$$

Spare Capacity Requirements:

$$
\begin{array}{rlrl}
G^{(3)} & =Q^{(3)^{T}} M^{(3)}\left(P^{(3)} H_{2}^{3} H_{1}^{2}\right) & & \\
G^{(l)} & =Q^{(l)^{T}} M^{(l)} P^{(l)}, & & l \in\left\{1,2^{\prime}\right\} \\
S^{(l)} & =\operatorname{row}-\max G^{(l)}, & l \in\left\{1,2^{\prime}, 3\right\} \\
S^{(l)} \in \mathbb{R}^{\left|\mathcal{L}^{(l)}\right|}, & & l \in\left\{1,2^{\prime}, 3\right\}
\end{array}
$$

Path Disjointness:

$$
\begin{aligned}
P^{(1)}+Q^{(1)} & \preceq 1 \\
P^{\left(2^{\prime}\right)}+Q^{\left(2^{\prime}\right)} & \preceq 1 \\
\left(P^{(3)}+Q^{(3)}\right) H_{2}^{3} H_{1}^{2} & \preceq 1
\end{aligned}
$$

The JCA $3 \mathrm{~L}$ problem has the objective to jointly minimize the total capacity requirement at the WDM layer due to flows at this and the other two layers. This is given in (5). Then there are four blocks of constraints. The first block deals with the basic requirements of the primary paths in each of the three layers. Constraint (6) is a flow conservation constraint, guaranteeing no traffic lost between two end-points. Constraint (7) calculates working capacity requirements from a perspective of the $l$-th layer. Constraints (8)-(9) define variables with respect to the primary paths.

The second block of constraints deals with backup paths. Constraint (10) defines the flow conservation constraint for the backup paths in Layer $l$, where $l=2^{\prime}$ refers to Layer 2 traffic from the perspective of Layer 1 . In other words, this Layer 2 traffic is mapped onto Layer 1. For example, in this case, $Q^{\left(2^{\prime}\right)}$ is a backup path of Layer 2 traffic provided at Layer 1 which is denoted as $2^{\prime}$. Constraint (11) defines variables with respect to this second block of constraints.

The third block of constraints considers the spare capacity requirements of the backup paths. Constraint (12) determines the amount of capacity units needed at the WDM layer in order to protect the primary overlay paths $P^{(3)}$ upon their failures. The last component in this constraint translates the capacity requirement to the view of the WDM layer using crosslayer mappings $(H \mathrm{~s})$ that conform with Theorem 1 . At the IP layer, the situation is a little different. This is because while primary paths are calculated at this layer, the backup paths are provided at the WDM layer. We chose this survivability strategy for IP traffic by means of the best strategy as studied in [15]. Since traffic at the WDM layer is also provided backup paths at the WDM layer, this requires no translation. Therefore, whether traffic originates at the IP or the WDM layer, the amount of capacity units can be determined by constraint (13). The row-max (.) operation in (14) determines the maximum element in each row of the matrix $G^{(l)}$. These elements reflect the amount of capacity needed by their corresponding link in order to protect against a single link failure at the WDM layer. Like previously given, constraint (15) defines a variable 


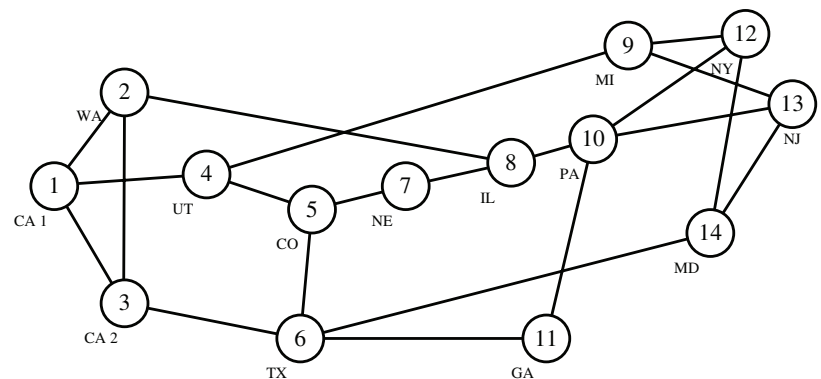

Fig. 2. The 14-node, 21-link NSFNet

with respect to spare capacity of the backup paths. These spare capacity matrices are not allowed to share across layers; however, the capacity can be shared within layer.

The relationship between the primary and backup paths are provided in the fourth block of constraints. Constraint (16) guarantees that, at Layer 1, each backup path matrix is disjoint from the primary paths. Constraint (17) guarantees paths disjointness of Layer 2 traffic at Layer 1. As the backup path to Layer 2 traffic flows is provided at Layer 1, this constraint guarantees such disjointness between this backup path and the mapped primary path. At Layer 3, both primary and backup paths are computed at this layer; however, because the overlay network has no knowledge of the underlying layers, they are not necessarily disjoint from the perspective of Layer 1. Constraint (18) guarantees such disjointness at Layer 1. The JCA3L problem is NP-hard as it can be reduced from some known NP-hard problems [14], [20].

\section{Simulation Results AND Analysis}

In this section, we evaluate the efficiency and effectiveness of the crosslayer survivable mapping (CSM) in three-layer networks, we consider the NSFNet consisting of 14 nodes, 21 links, depicted in Fig. 2, as a Layer 1 (WDM) network. In the case of Layer 2 (IP) network, links are added to the NSFNet such that the network is fully connected. Layer 3 (overlay) network is constructed as a partial, i.e., half, mesh of the full mesh Layer 2 topology, as shown in Fig. 3. In here, the maximum number of overlay traffic flows in where each flow request is represented by an overlay link is 46 . These particular links are employed as a means to overcome crosslayer survivable mapping limitation in this topology that not all disjoint paths in the overlay layer are also disjoint at the WDM layer. It should be noted here that the considered single-link flows can easily be extended into the case when the flows require two or more overlay links by concatenating the links. In addition, we consider a number of demand flows at the overlay layer and a fixed number of demand flows at the IP and WDM layer; the numbers of IP and WDM traffic flows do not affect capacity requirements of the overlay since sharing spare capacity across layers is not allowed. Following the setting of a three-layer network, we present some results, provide discussions, and evaluate the performance of crosslayer survivable mapping against three other mappings in terms of capacity overbuilding,

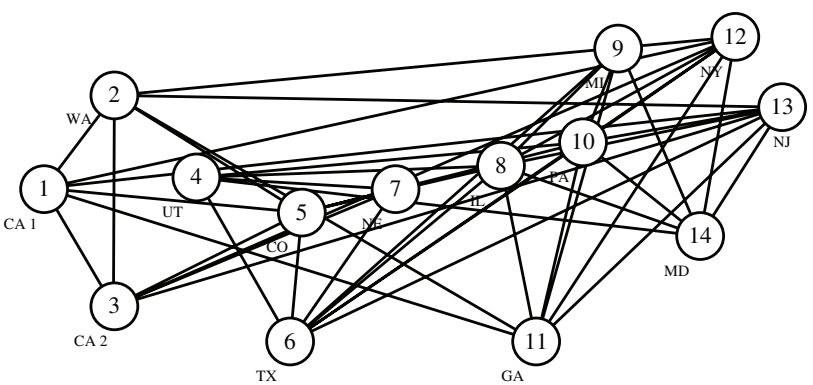

Fig. 3. The 14-node, 46-link overlay network

flow overrouting, and link overusing due to both primary and backup paths.

These three mappings are obtained by an alteration in one or both matrices of CSM: $\left(H_{1}^{2}, H_{2}^{3}\right)$ which conform to Theorem 1. The first mapping, M1: $\left(H_{1}^{2}, H_{2}^{3}\right)$, makes a change only in $H_{1}^{2}$ while the second mapping, M2: $\left(H_{1}^{2}, \widetilde{H_{2}^{3}}\right)$, makes a change in $H_{2}^{3}$ but not $H_{1}^{2}$. Finally, the third mapping, M3: $\left(\widetilde{H_{1}^{2}}, \widetilde{H_{2}^{3}}\right)$, makes a change in both of the matrices. In all cases, change is done in a way that only the corresponding two layers are involved in the design, i.e., backhaul routing is not being considered but each of the two-layer mappings is still survivable under single-link failures.

\section{A. Capacity Overbuilding}

Capacity overbuilding (CO), the percentage of the excess capacity requirement of the overlay traffic due to backhaul routing, is here used to evaluate the efficiency of CSM. The excess capacity occurs at the WDM layer whenever WDM links are unnecessarily used by overlay primary and/or backup paths. It should also be noted that $\mathrm{CO}$ is not the same as resource overbuild (RO) [21], which refers to a capacity ratio of backup paths to primary paths.

Figure 4 shows CO under various network loads that our proposed CSM can effectively eliminate the redundant capacity reservation. This is because it takes into account all three layers together by addressing the problem of backhaul routing. In here, the CSM has best CO partly because the designs of the two mappings - Layers 3 and 2, and Layers 2 and 1 - in all M1, M2, and M3 are carried out independently.

In addition, both $\mathrm{M} 1$ and $\mathrm{M} 2$ have better $\mathrm{CO}$ than M3 is because the alteration, discussed previously, has shown little change from the original mappings. This is partly due to the two-layer topology that has a limit on the number of mapping choices that a top-layer network has to be survivable under a single link failure at the lower layer. This limitation also happens in any two-layer network, but in the case of M1 versus M2 the situation is different. Their COs are dependent on topology, mapping, and order of flows. In this particular network M2 is outperformed by M1, in part for the two mapping matrices of the three-layer network and partly because full-mesh characteristics in Layer 2 (IP) network is said to provide much deviation in $\widetilde{H_{2}^{3}}$ from $H_{2}^{3}$ in terms of element-wise comparison of the two matrices. The COs of the 


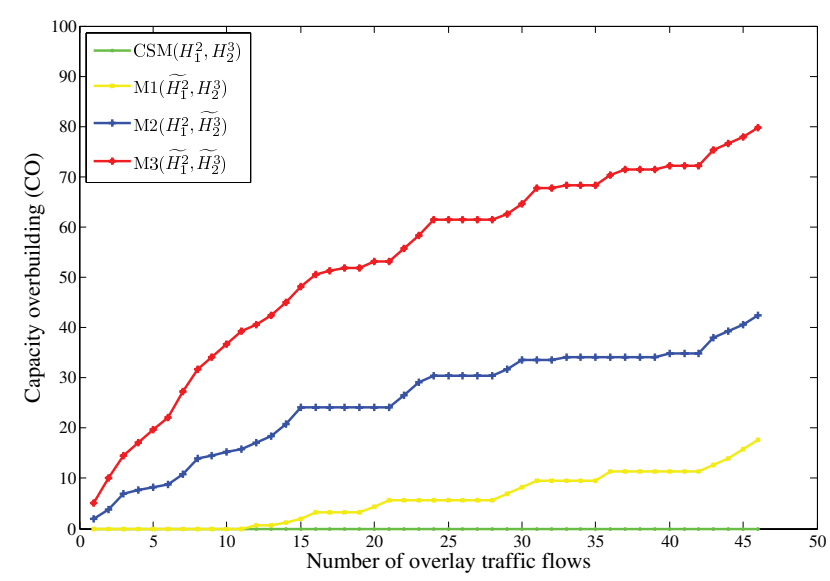

Fig. 4. $\mathrm{CO}$ of the overlay network

four schemes become more obviously different under a high number of overlay traffic flows because backhaul routes occur more.

\section{B. Flow Overrouting}

Defined as the ratio of the number of overlay traffic flows that are routed on one backhaul link or more at the WDM layer to the total number of the overlay flows, flow overrouting (FO) is used to show the number of overlay flows that are affected by backhaul routing. In a similar manner to CO, Fig. 5 shows that CSM can solve the problem while some flows in M1, M2, or M3 still suffer from it. In all M's, the flat FO at the beginning is understood to coincident with the flows ordering in our studied network; however, an average number of the affected flows is steady as the number of overlay traffic flows is increasing. In particular when the number of flows is 11 or fewer, M1 has no flow over routed, i.e., 0\% FO. This corresponds to its $\mathrm{CO}$ in Fig. 4 that M1 also has no capacity overbuilt when there are 11 flows or fewer. In contrary to M1, M2 has a $100 \%$ FO when there are 15 flows or fewer. This is because neither of the primary nor backup paths are overrouted. This observation of the FO again corresponds to the $\mathrm{CO}$ in Fig. 4 but with a different degree when the number of flows varies between 1 to 15 .

\section{Link Overusing}

Link overusing (LO) is used to show unnecessary use of the WDM links. LO is defined as the ratio of backhaul routing happens at the WDM layer that affect overlay traffic flows to the total number of WDM links that are used by the overlay. This can be regarded as FO from the viewpoint of the WDM layer, instead of the overlay. In the cases of M2 and M3, it is shown in Fig. 5 that at the beginning, e.g., fewer than 10 flows, every overlay flow is affected by backhaul routing; but when we look at LO, Fig. 6 shows that only some of the WDM links are unnecessarily used more than once. This is the same problem discussed earlier and shown in Fig. 1 that capacity, or wavelength, saving in WDM links can be accomplished by

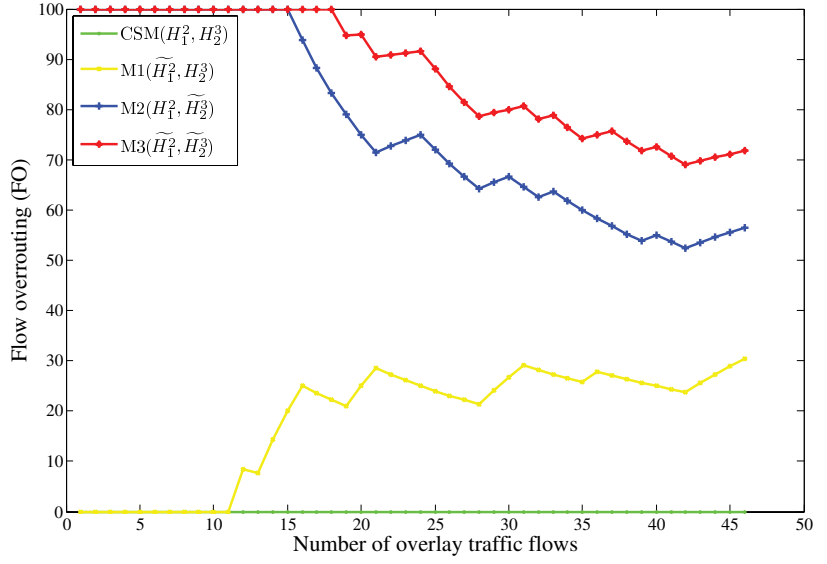

Fig. 5. FO of the overlay network

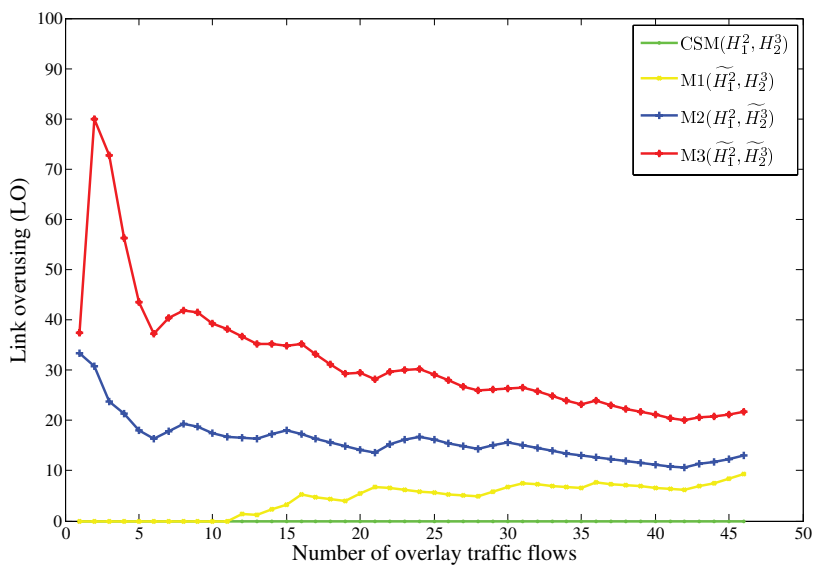

Fig. 6. LO of the overlay network

letting an overlay flow use only some WDM links rather than these links and some other links more than once. A similar argument can also be applied to M1 with an interesting case at the beginning when the number of flows is 11 or fewer: We witness a unanimous correspondence among the LO, FO, and $\mathrm{CO}$ here in the sample network that backhaul routing does not occur for the first 11 flows in the case of M1; therefore, no capacity is overbuilt.

\section{CONCLUSIONS}

We are moving into a new era of three-layer networks. Survivability network designing in the planning phase is sophisticated but from this paper, it is clear that each layer should not be designed separately. We show that a complete picture of the network is essential for efficient capacity allocation and the survivability of services in all three network layers. Ironically enough, the dependency of layers, which until now has largely been considered as an insignificant issue, is shown to be extremely important, even when a backup path is provided.

The concept of crosslayer survivable mapping, which consider link mapping across all layers, introduced in this paper 
is applicable to all multilayer networks and is highly useful and particularly necessary for a network of two layers or more. Simulation results confirm that our design approach can overcome the inefficiency of capacity allocation and at the same time can also guarantee an overlay that it be survivable under any single-link failure at the two layers below compared to existing approaches of designing two mappings independently.

This work advances our understanding of the structure of multilayer networks and can be extended for a number of promising directions for further research including multilayer routing and differentiated service guarantees, when some mapping choices are more robust than the others.

\section{REFERENCES}

[1] A. Markopoulou, G. Iannaccone, S. Bhattacharyya, C.-N. Chuah, Y. Ganjali, and C. Diot, "Characterization of failures in an operational IP backbone network," IEEE/ACM Trans. Netw., vol. 16, no. 4, pp. 749762,2008

[2] R. Ramaswami and K. Sivarajan, Optical Networks: A Practical Perspective (Second Edition) (The Morgan Kaufmann Series in Networking), 2nd ed. Morgan Kaufmann, 102001.

[3] D. Medhi and D. Tipper, "Multi-layered network survivability-models, analysis, architecture,framework and implementation: an overview," in DARPA Information Survivability Conference and Exposition, 2000. DISCEX '00. Proceedings, vol. 1, Hilton Head, SC, USA, 2000, pp. 173-186.

[4] M. Pickavet, P. Demeester, D. Colle, D. Staessens, B. Puype, L. Depre, and I. Lievens, "Recovery in multilayer optical networks," Journal of Lightwave Technology, vol. 24, no. 1, pp. 122-134, Jan. 2006.

[5] B. Puype, J. P. Vasseur, A. Groebbens, S. De Maesschalck, D. Colle, I. Lievens, M. Pickavet, and P. Demeester, "Benefits of GMPLS for multilayer recovery," IEEE Communications Magazine, vol. 43, no. 7, pp. 51-59, Jul. 2005.

[6] M. Vigoureux, B. Berde, L. Andersson, T. Cinkler, L. Levrau, M. Ondata, D. Colle, J. Fernandez-Palacios, and M. Jager, "Multilayer traffic engineering for GMPLS-enabled networks," IEEE Communications Magazine, vol. 43, no. 7, pp. 44-50, Jul. 2005.

[7] F. Touvet and D. Harle, "Network resilience in multilayer networks: A critical review and open issues," in ICN '01: Proceedings of the First International Conference on Networking-Part 1. London, UK: SpringerVerlag, 2001, pp. 829-838.
[8] B. Rajagopalan, J. Luciani, and D. Awduche, "IP over Optical Networks: A Framework," RFC 3717 (Informational), Mar. 2004

[9] J.-P. Vasseur, M. Pickavet, and P. Demeester, Network Recovery: Protection and Restoration of Optical, SONET-SDH, IP, and MPLS (The Morgan Kaufmann Series in Networking). Morgan Kaufmann, 72004.

[10] S. D. Madhu Kumar and U. Bellur, "Availability models for underlay aware overlay networks," in DEBS '08: Proceedings of the second international conference on Distributed event-based systems. New York, NY, USA: ACM, 2008, pp. 169-180.

[11] D. Andersen, H. Balakrishnan, F. Kaashoek, and R. Morris, "Resilient overlay networks," in SOSP '01: Proceedings of the eighteenth ACM symposium on Operating systems principles. New York, NY, USA: ACM, 2001, pp. 131-145.

[12] P. Demeester, M. Gryseels, A. Autenrieth, C. Brianza, L. Castagna, G. Signorelli, R. Clemenfe, M. Ravera, A. Jajszczyk, D. Janukowicz, K. Van Doorselaere, and Y. Harada, "Resilience in multilayer networks," IEEE Communications Magazine, vol. 37, no. 8, pp. 70-76, Aug. 1999.

[13] A. Urra, E. Calle, and J. L. Marzo, "Multi-layer network recovery: Avoiding traffic disruptions against fiber failures," in Computational Science ICCS 2006, ser. LNCS, vol. 3994 . Springer-Verlag Berlin / Heidelberg, 2006, pp. 136-143.

[14] E. Modiano and A. Narula-Tam, "Survivable routing of logical topologies in WDM networks," in Proc. IEEE INFOCOM 2001., vol. 1, Anchorage, AK, Apr. 2001, pp. 348-357.

[15] P. Pacharintanakul and D. Tipper, "The effects of Multi-Layer traffic on the survivability of IP-over-WDM networks," in Proc. IEEE ICC 2009., Dresden, Germany, Jun 2009.

[16] R. K. Ahuja, T. L. Magnanti, and J. B. Orlin, Network Flows: Theory Algorithms, and Applications. Prentice Hall, 1993.

[17] Y. Liu, D. Tipper, and K. Vajanapoom, "Spare capacity allocation in two-layer networks," IEEE JSAC, vol. 25, pp. 974-986, Jun. 2007.

[18] K. Lee and E. Modiano, "Cross-layer survivability in WDM networks with multiple failures," in OFC/NFOEC 2008. Conference on, San Diego, CA, Feb. 2008, pp. 1-3.

[19] - "Cross-layer survivability in WDM-based networks," in Proc. IEEE INFOCOM 2009., vol. 1, Rio de Janeiro, Brazil., Apr. 2009., pp. $1017-1025$.

[20] M. R. Garey and D. S. Johnson, Computers and Intractability: A Guide to the Theory of NP-Completeness. New York, NY, USA: W. H. Freeman \& Co., 1979.

[21] L. Song and B. Mukherjee, "New approaches for dynamic routing with availability guarantee for differentiated services in survivable mesh networks: The roles of primary-backup link sharing and multiple backup paths," in Proc. IEEE GLOBECOM 2006., San Francisco, CA, Nov./Dec. 2006, pp. 1-5. 